\title{
Adaptation to Knee Flexion Torque During Gait
}

\author{
James S. Sulzer, Keith E. Gordon, T. George Hornby, Michael A. Peshkin and James L. Patton
}

\begin{abstract}
Studies have shown locomotor adaptation of the ankle to assistive torques, but the ability of the knee to adapt to assistive forces has not yet been explored. Understanding how humans modulate knee joint kinematics during gait could be valuable for designing assistive devices for stroke patients. In this study we examined how healthy subjects adapt to knee flexion torque assistance during gait. We hypothesized that 1) when given assistance subjects would adapt their locomotor patterns to walk with kinematics similar to baseline values and 2) removal of assistance following adaptation would result in substantially reduced knee flexion. Contrary to expectations, data from the five subjects show a weak adaptation and an increase in knee flexion kinematics after forces were removed. These results suggest that neuromuscular control of the knee joint during walking is not strongly modulated by feedforward mechanisms.
\end{abstract}

\section{INTRODUCTION}

The use of lower limb exoskeletons to enhance and restore movement ability is becoming abundant. There are competing philosophies about designing such assistive devices. The first approach aims to create multipurpose (i.e. walking, standing) devices by actuating many lower limb joints [1]. Alternatively, by targeting specific functions, light weight, affordable exoskeletons can be designed that minimize actuator number and power consumption [2]. This minimal assistance philosophy may be valuable for assisting specific gait deficits experienced by individuals with neurological disorders.

Many stroke survivors will suffer from a specific gait disability called Stiff-Knee Gait (SKG). SKG is characterized by reduced knee flexion during the swing phase. While unable to bend their knee, people with SKG typically have some residual strength and control of their affected limb. With a human system already partially capable of actuation, it would be illogical to assume that a full lower-body exoskeleton would be necessary to restore healthy gait. Our goal is to use a robotic intervention to determine the role of knee activity in SKG. Insights from this study may be

This work was supported by the U.S. Department of Veterans Affairs

J. Sulzer is with the Laboratory for Intelligent Mechanical Systems at Northwestern University and the Sensory Motor Performance Program at the Rehabilitation Institute of Chicago, Chicago, IL 60611, USA James Sulzer2009@u.northwestern.edu

K. Gordon is with the Sensory Motor Performance Program, Rehabilitation Institute of Chicago, Chicago, IL 60611, USA, keith-gordonenorthwestern. edu

T. G. Hornby is with the faculty of Physical Therapy at the University of Illinois-Chicago and the Sensory Motor Performance Program at the Rehabilitation Institute of Chicago, Chicago, IL 60611, USA, tgh@uic. edu

M. Peshkin is with the faculty of Mechanical Engineering at Northwestern University, Evanston, IL 60201, USA peshkin@northwestern. edu

J. Patton is with the faculty of Bioengineering at University of IllinoisChicago and the Sensory Motor Performance Program at the Rehabilitation Institute of Chicago, Chicago, IL 60611, USA patton jeuic.edu valuable in the development of an efficient, lightweight and affordable assistive device for SKG.

The minimal assistance philosophy can be extended past joint space to time. For instance, modeling studies have indicated that knee flexion activity during double support and knee flexion velocity at foot off are directly related to increased knee flexion during the swing phase [3], [4], [5]. Therefore to increase knee flexion angle during swing, one should only need to actuate during double support. We have developed a lightweight, transparent, powered knee orthosis for the purpose of selectively assisting knee flexion torque during double support [6]. The novelty of this device is that it is capable of exerting pure torque (without an associated impedance), due to the extreme compliance of the spring used in the series elastic mechanism.

In this study, we examine the effect of providing assistive knee flexion torque immediately prior to foot off on neurologically intact subjects with the goal of eventually comparing to a stroke population. Similar to a previous adaptation study [8], we hypothesized that repeatedly providing knee flexion torque assistance directly before swing would initially increase knee flexion angle during swing, but gradually diminish towards baseline during training due to adaptation. Following training, we predicted subjects would initially decrease knee flexion and then slowly return to baseline values due to after-effects of the adaptive process. We predicted the same relationship for knee flexion velocity at foot off and maximum toe height. Such an adaptation should imitate the weakness that is hypothesized to be the cause of SKG in the stroke population. We speculated that these after-effects in the healthy subjects would bring about the secondary effects associated with SKG in stroke.

In this experiment, we find that subjects have a weak adaptation to the assistance. It appears from the kinematic data that some subjects may be resisting knee flexion assistance instead of allowing the device to create knee flexion on its own. This finding suggests that the knee is not an active source of positive power during gait in healthy subjects.

\section{METHODS}

Five healthy subjects ( 2 female), age (mean \pm SD) $25.6 \pm$ 3.8 years, and weight $74.9 \pm 16.2 \mathrm{~kg}$ provided informed consent to a protocol approved by the Institutional Review Board of Northwestern University and participated in the study. Each subject walked on an instrumented split-belt treadmill (ADAL, Tecmachine, Andrezieux Boutheon, France). We used real-time motion capture cameras (Motion Analysis Corporation, Santa Rosa, CA) to record reflective markers 


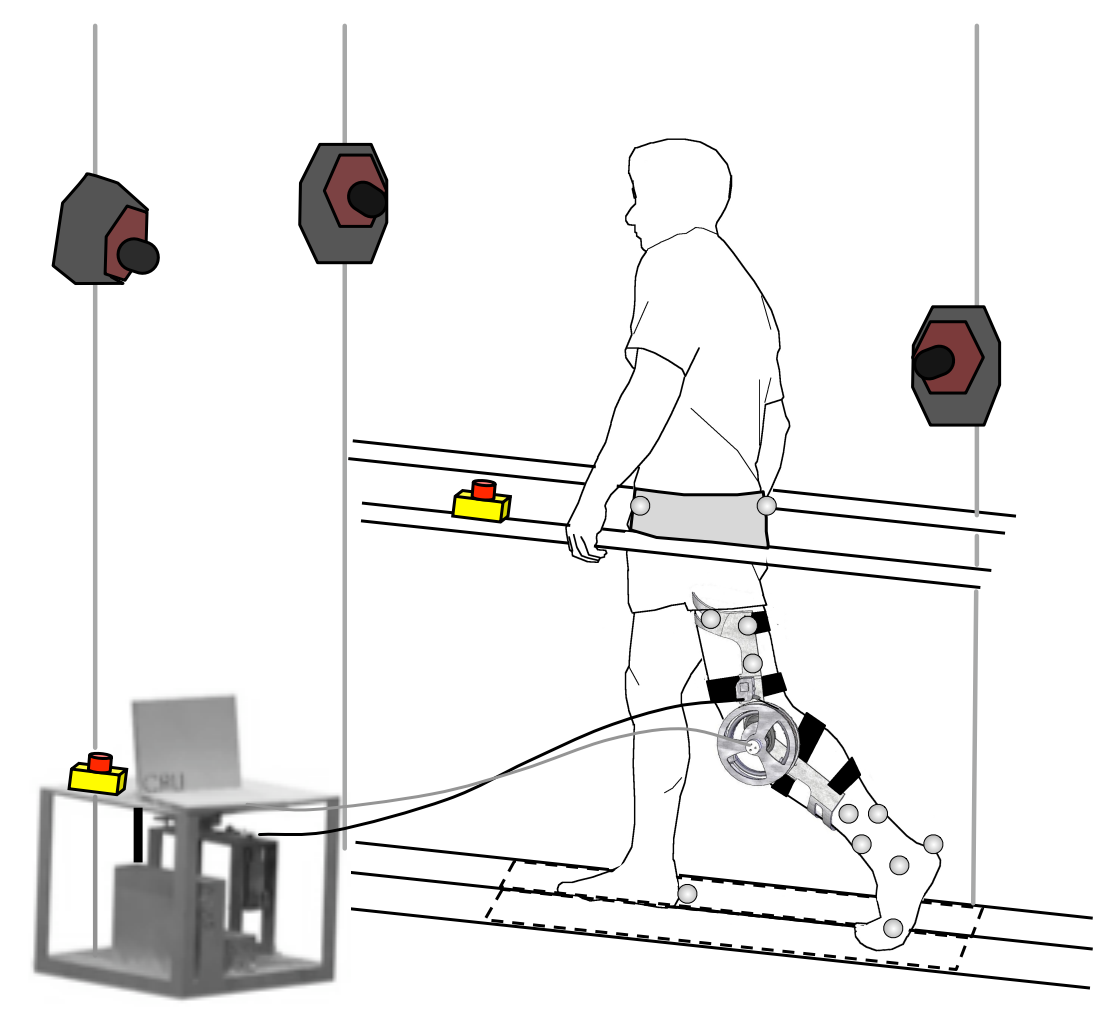

Fig. 1. Experimental setup includes an instrumented, split-belt treadmill, a motion capture system, and a tethered powered orthosis (Series Elastic Remote Knee Actuator - SERKA) applying selective knee flexion torque during double support.

located on bony landmarks (Figure 1) at $100 \mathrm{~Hz}$. Emergency stop switches for both user and experimenter were provided.

A powered knee brace donned unilaterally by each subject (Figure 2) was developed to selectively apply knee flexion torque during a specified interval, and then remain transparent for the remainder of the gait cycle. The device itself is composed of a modified commercial knee brace (Bledsoe Axiom Sport Hi-Impact). The brace only allows knee flexion/extension, and has a hard stop to prevent knee hyperextension. Torque is supplied to the brace by a servomotor $(1.4 \mathrm{~kW})$ with a 5:1 gear reduction remotely located away from the user (Figure 1). The device is capable of supplying up to $41 \mathrm{~N} \cdot \mathrm{m}$ of torque and is capable of creating a substantial $(12 \mathrm{~N} \cdot \mathrm{m})$ torque in under $20 \mathrm{~ms}$. In addition, the powered orthosis is almost imperceptible when not active, resisting with an average torque of $0.67 \mathrm{~N} \cdot \mathrm{m}$. The weight of the device on the user is $1.2 \mathrm{~kg}$. The motor is controlled by a servoamplifier and PC controller (PC104 stack) using Matlab XPC at $1 \mathrm{kHz}$. The design and performance has been described in detail [6].

After being fit with the knee brace and reflective markers, subjects walked with the device during an evaluation period. The evaluation was performed to determine the level of torque to be used during training. Each subject walked at a slow speed $(2.0 \mathrm{~km} / \mathrm{h})$ while knee flexion torque was applied during the pre-swing phase of pseudorandomly selected steps. Subjects were informed only that the device would "assist knee flexion". We detected gait phase using the load cells in the instrumented treadmill. Activation occurred 200 ms before foot off. The device predicted foot-off time based on the previous step. We graded the flexion torque assistance to rise each cycle until the knee achieved a knee flexion angle during swing $20^{\circ}$ greater than baseline. The evaluation period lasted 50 steps.

The next stage was composed of two blocks, each 680 steps in duration. The first block was conducted at a speed of $2.0 \mathrm{~km} / \mathrm{h}$, and the second block at $4.0 \mathrm{~km} / \mathrm{h}$. Speeds were chosen based on typical walking speed for stroke [7], and average walking speed for healthy subjects. Each block was composed of three phases: baseline (20 steps) when no torque was exerted, training (560 steps) when the torque determined from the evaluation period was provided, except during four pseudorandomly placed catch trials, and washout (100 steps) where assistance was removed immediately following training.

We recorded four consecutive steps of data at 20 different intervals (one at end of baseline, one at beginning of training, 16 pseudorandomly during training, one each at beginning, middle and end of washout), recording device torque, load cells and kinematics. After the experiment, data were low pass filtered using a 5th order Butterworth filter at $8 \mathrm{~Hz}$. We examined knee flexion angle, knee flexion velocity, and maximum toe height during gait.

Since markers on the thigh were attached to the brace, 


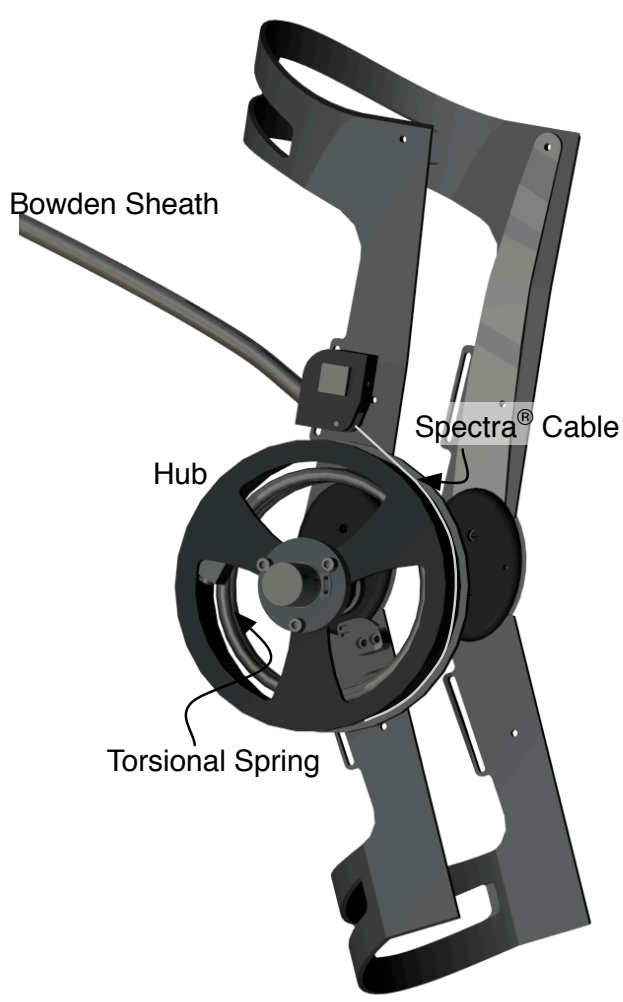

Commercial Knee Brace

Fig. 2. The structure of the SERKA is composed of a torsional spring anchored to a commercial knee brace, with the opposite end of the spring attached to an aluminum hub. The deflection of the hub relative to the brace translates to torque through the spring, and is controlled by the excursion of Spectra cable passing through a Bowden sheath.

knee angle calculated from marker data would reflect the displacement of the knee brace instead of the knee. To accommodate for this, we calculated thigh position by first calculating the center of rotation of the hip relative to the pelvis, and then the knee relative to the shank.

\section{RESULTS}

Knee flexion assistance increased knee flexion angle, with smaller effects at the hip and ankle. Figure 3 shows the mean sagittal plane kinematics of the hip, knee and ankle averaged over all five subjects. From baseline to initial exposure, knee flexion during swing increased $20^{\circ}$ for both slow and typical walking cases. For the slow case, knee flexion angle during swing remained the same throughout training, but in the typical walking case, decreased $5^{\circ}$. After training, knee kinematics returned to baseline patterns.

We observed the same relationship with peak hip flexion, but to a smaller extent. In both slow and typical cases, mean peak hip flexion during swing increased $5^{\circ}$ during assistance. After assistance, hip flexion returned to baseline values for the typical case, but remained higher than baseline after training.

Ankle kinematics also alter during the swing phase. Although there is no difference from baseline to initial exposure, as training persists, we observed greater mean ankle plantarflexion during swing in both cases $\left(3^{\circ}\right)$. In the slow case, this plantarflexion continued into washout.

The bottom of the figure shows the average torque profile exerted on the knee. The profile rises to its maximum value within $75 \mathrm{~ms}$, taking a smooth, sigmoidal path. Once foot off is detected, the torque reduces back to near zero torque, again using a smooth trajectory.

Exposure to forces for the duration of the training period resulted in some adaptation. For both the slow and typical walking speeds (Figure 4), maximum knee flexion angle increased over $20 \%$ from baseline (significantly greater at $p<0.001$, t-test). Catch trials at both speeds averaged less than baseline, but with no statistical significance. During washout, knee flexion decreased significantly compared to baseline $(p<0.001)$ in both cases, and then increased over time, but only significantly for the slow walking case $(p<0.001)$.

According to modeling studies, higher knee flexion velocity at foot off should result in increased knee flexion angle during swing. In Figure 5, knee flexion velocity at foot off immediately increased by an average of $80 \%$ before settling at nearly a $40 \%$ increase. The rise in knee flexion velocity relative to baseline was significantly greater than the increase in knee flexion angle in the slow case $(p<0.001)$, and nearly significant in the typical walking speed $(p=0.059)$. As in Figure 4, catch trials averaged below zero, but with only one trial (last catch trial of the typical walking speed) significantly below baseline. Washout was also similar, with an initial drop near baseline once the forces are removed, and then increased afterwards. However, the only significant differences from baseline in washout were seen in the slow case $(p<0.02)$.

Maximum toe height during walking can also be used as a functional kinematic measure. Figure 6 shows that average maximum toe height in both cases initially increased near $30 \%$ of baseline, and then fell near or under $20 \%$ increase. This increase compared to knee flexion angle increase was not significant. While the increase from baseline is significant $(p<0.001)$, the parameter did not significantly change from initial exposure by the end of training. Catch trials again averaged below baseline with one trial in both speeds significantly below ( $p<0.01$ for slow, $p<0.02$ for typical).

\section{DISCUSSION AND CONCLUSIONS}

\section{A. Discussion}

As predicted, primary outcome measures such as maximum knee flexion, knee flexion velocity at foot off, and maximum toe height all increased when subjects received knee flexion assistance prior to foot off. Results from the catch trials indicated that subjects adapted.

While knee flexion increased on average $30 \%$, knee flexion velocity at foot off increased at a much greater rate. A modeling study by Goldberg et al. [3] simulated knee perturbations and found knee flexion velocity at foot off to be approximately linear with knee flexion angle during swing. This discrepancy would indicate that the user may be using some effort to slow the knee down instead of allowing 

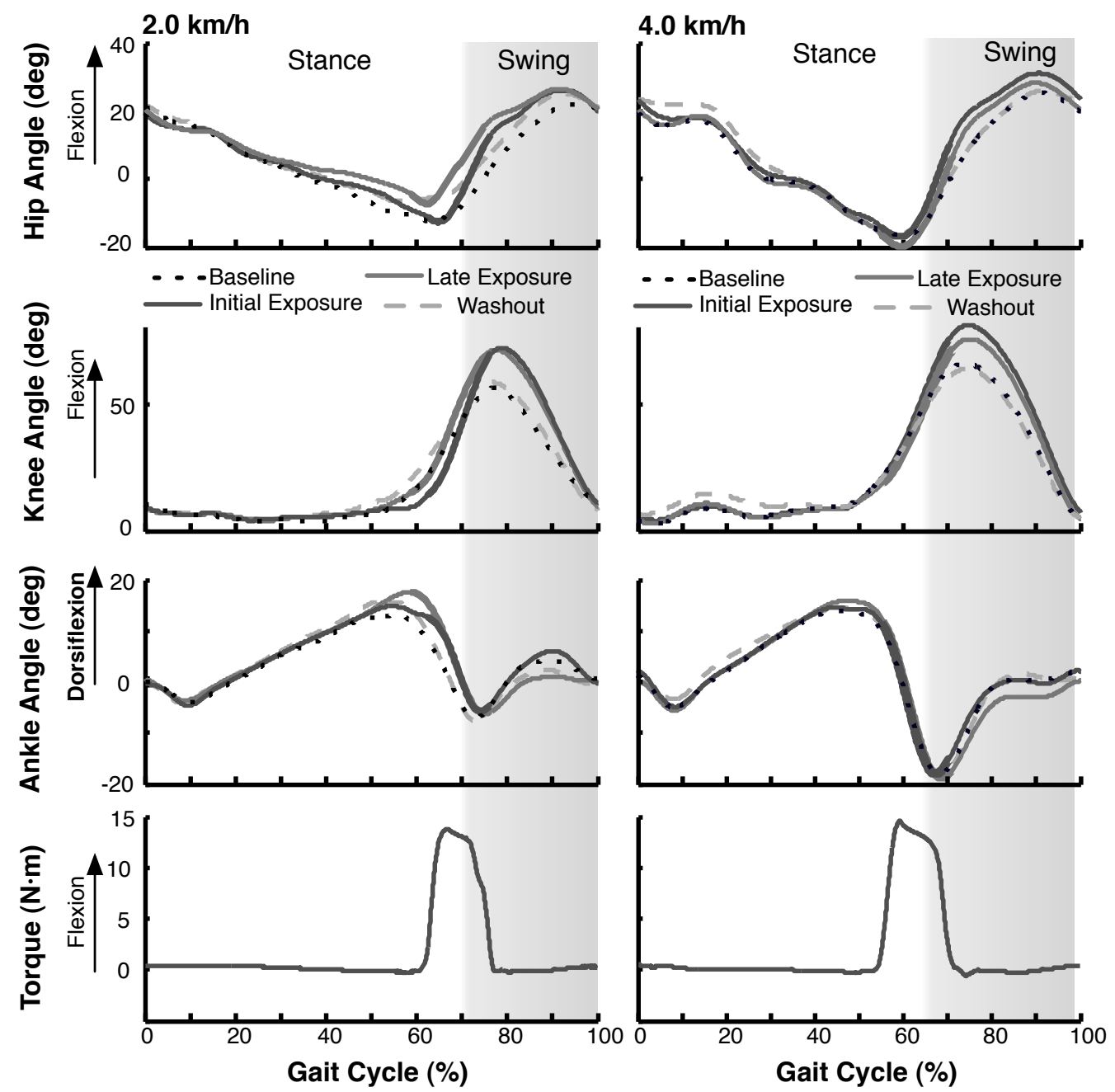

Fig. 3. Sagittal plane kinematics of the hip, knee and ankle against percent gait cycle at four different stages: baseline, initial exposure, late exposure and initial washout. The data are averaged over all subjects. Baseline and washout are shown as dark dotted and light dashed lines, respectively. Initial and late exposure are shown as dark and light solid lines, respectively. The period of assistance is represented in two ways: first, where initial and late exposure data becomes thicker, and second, on the bottom subplot where a sample torque pulse is shown.

the device to move their knee. If this is true, then it would be consistent with modeling studies that treat the knee as a brake (although in this case, the brake is in extension), needing little to no assistance from knee flexors to bend [9].

The washout stage was far different than expected. Our expectations were reduced knee flexion angle immediately following training, inducing gait characteristic of SKG in a healthy population. However, instead of observing less knee flexion after assistance as a result of adaptation, we observed a rise in knee flexion angle. This same effect was echoed in knee flexion velocity at foot off. Indeed, three of the subjects claimed their leg felt "lighter" during washout, contrary to our expectations. However, given our observation that the quadriceps appear to be reducing knee flexion velocity after foot off, the gradual reduction in abnormal quadriceps activity would help explain this result.

\section{B. Conclusions and Future Work}

In summary, knee flexion torque during the double support phase increased knee flexion angle during swing, and subjects exhibited few signs of adaptation. It appears that subjects do not adjust their gait back to baseline during 
training, which could indicate that knee flexion angle is not a highly controlled parameter in pre-swing and swing phase of gait. From these results, we would not expect large adaptations in subjects with SKG either, which will be examined in future work.

\section{ACKNOWLEDGMENTS}

The authors gratefully acknowledge the contributions of Yasin Dhaher, Ph.D., Dr. Ross Bogey, and the U.S. Department of Veterans Affairs.

\section{REFERENCES}

[1] Suzuki, K., Y. Kawamura, T. Hayashi, T. Sakurai, Y. Hasegawa, and Y. Sankai. Intention-based walking support for paraplegia patient. IEEE International Conference on Systems, Man and Cybernetics. Oct. 2005, 2707-13.

[2] Walsh, C. J., D. Paluska, K. Pasch, W. Grand, A. Valiente, H. Herr. Development of a lightweight, underactuated exoskeleton for loadcarrying augmentation Proceedings of the 2006 IEEE International Conference on Robotics and Automation Orlando, Florida. May 2006 3485-91.

[3] Goldberg, S.R., F. A. Anderson, M. G. Pandy, S.L. Delp, Muscles that influence knee flexion velocity in double support: implications for stiff-knee gait. Journal of Biomechanics, 2004. 37: p. 1189-1196.

[4] Anderson, F.C., et al., Contributions of muscle forces and foot off kinematics to peak knee flexion during the swing phase of normal gait: an induced position analysis. J Biomechanics, 2004. 37: p. 7317.

[5] Piazza, S.J. and S.L. Delp, The influence of muscles on knee flexion during the swing phase of gait. J Biomechanics, 1996. 29(6): p. 72333.

[6] Sulzer, J. S., R. A. Roiz, M. A. Peshkin and J. L. Patton. A highly backdrivable, lightweight knee actuator for investigating gait in stroke. IEEE Transactions on Robotics. (in press).

[7] Kerrigan, D. C., M. E. Karvosky, and P. O. Riley, Spastic paretic stifflegged gait: joint kinetics, Am J Phys Med Rehabil, vol. 80, no. 4, pp. 2449, 2001.

[8] Gordon, K., and D. Ferris. Learning to walk with a robotic exoskeleton. Journal of Biomechanics Volume 40, Issue 12, p. 2636-2644

[9] McGeer, T. Passive walking with knees. In Proceedings of the IEEE Robotics and Automation Conference. Los Alamitos, CA. p. 1640-45. 1990.

[10] Kerrigan, D.C. J. Gronley, and J. Perry. Stiff-Legged Gait in Spastic Paresis: A Study of Quadriceps and Hamstrings Muscle Activity. American Journal of Physical Medicine \& Rehabilitation. 70:294 1991
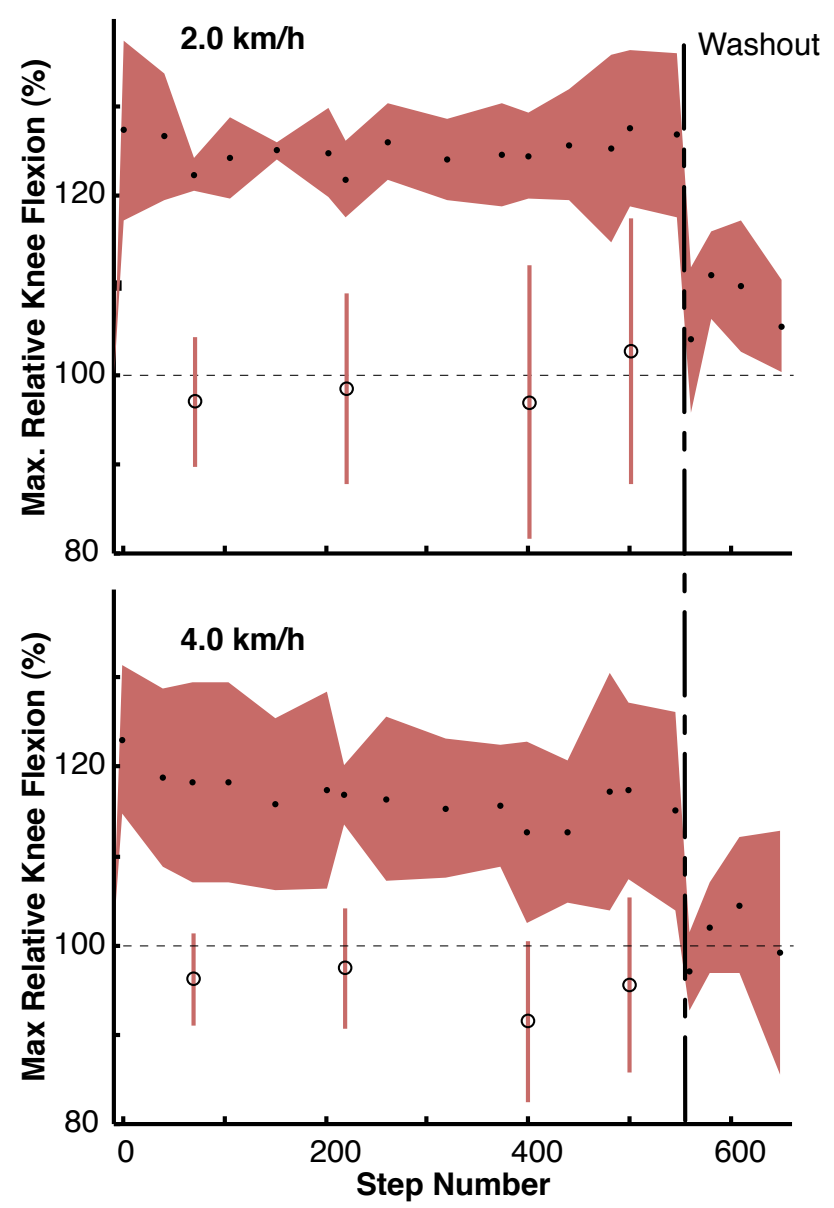

Fig. 4. Maximum knee flexion during training and washout, displayed relative to baseline. Average knee flexion over the five subjects is shown in black dots, (catch trials shown in black circles), where the red region shows 95\% confidence interval. 


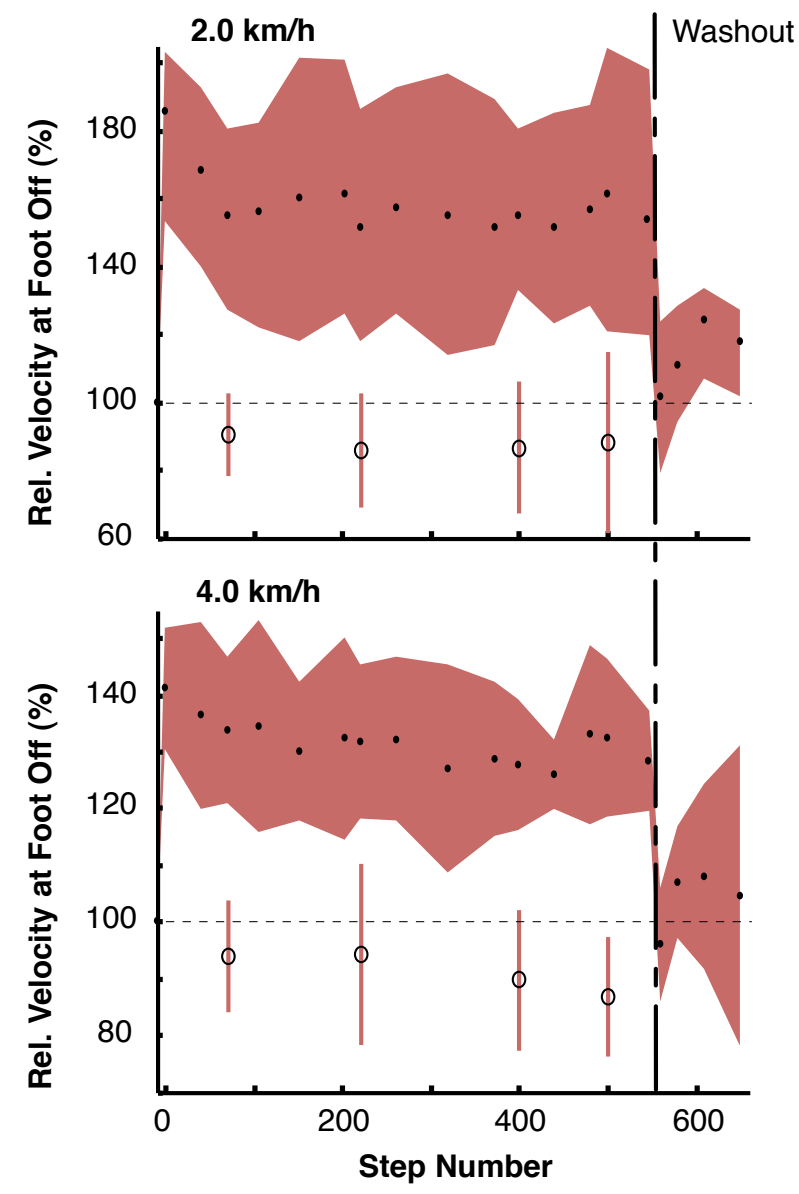

Fig. 5. Knee flexion velocity at foot off similarly reflects the relationships in Figure 4 as expected.

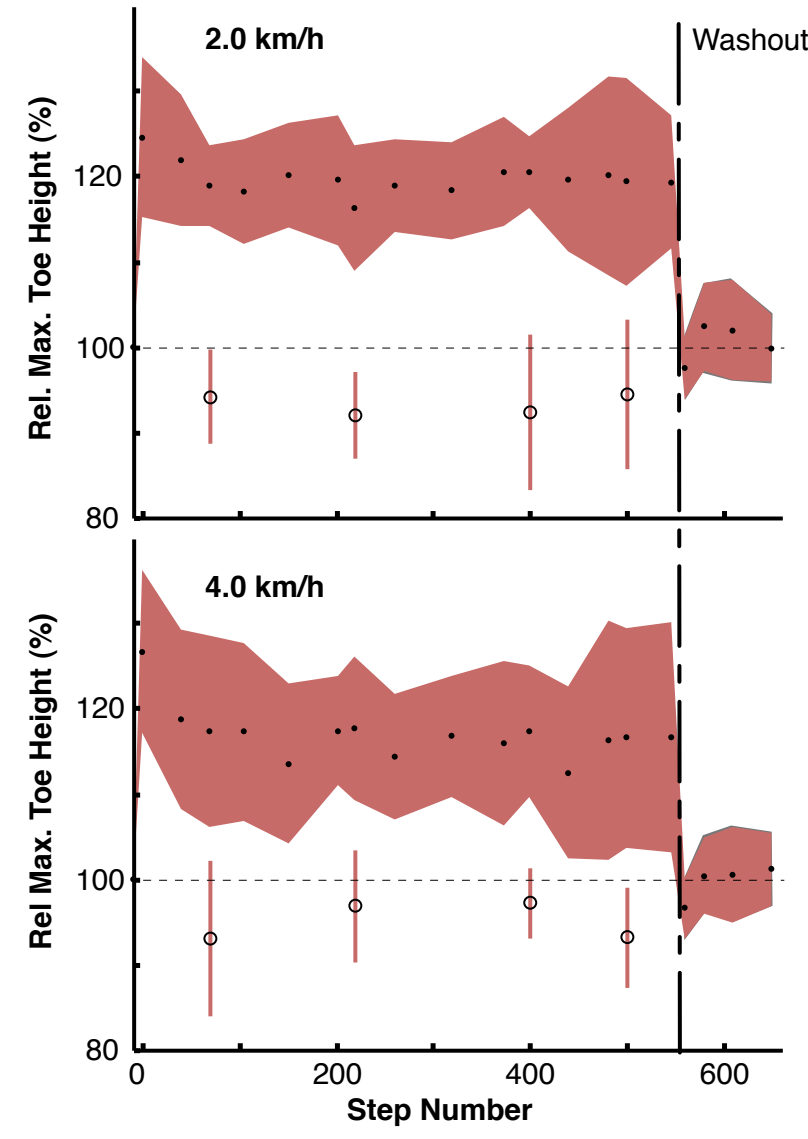

Fig. 6. Maximum toe height follows the patterns of maximum knee flexion angle, also as expected. Note there are catch trials significantly below baseline for both fast and slow cases, indicating some adaptation. 